\title{
Transmission of Wellness Information Signals Using an Inerton Field Channel
}

\author{
Volodymyr Krasnoholovets, Vasyl Fedorivsky
}

\section{ABSTRACT}

We examine the nature of field signals capable to correct the functioning of the human body and the possibility to transmit such signals from a remote source. To understand this, the matter wave (i.e. the particle's wave $\psi$ function) is expanded in two subsystems: the particle itself and a cloud of spatial excitations named inertons. They are inertons that carry fragments of mass and provide a short-range action between adjacent atoms. The existence of inertons has already been confirmed in many experiments. A system of entities (atoms or molecules) is filled with their own inertons and such system has to be considered as a casing filled with an inerton gas. These inertons indeed behave like a gas that fluctuates at the same frequencies as the molecules of the casing. It was shown previously that inertons periodically come from the mass state $m$ to the tension state $\xi$. This means that inertons behave as a typical wave oscillating between the compression and stretching. Inerton wavelets are capable of being emitted from the system of vibrating entities. It is demonstrated that inerton wavelets can carry information to a remote receptor (distant even by many kilometres) transmitting to it the necessary information signals.

Keywords: phonons, inertons, signal trasnmission, information therapy.

\section{INTRODUCTION}

In recent decades, research in the field of information medicine has become widespread. These are approaches such as the Teslar watch method (generation of weak signals at $8 \mathrm{~Hz}$ ) [1]-[3]; method of microwave resonant therapy [4][6]; method of electro-acupuncture diagnostics by Reinhard Voll [7] with the addition of homeopathy-like remedies and various computer diagnostics that allow for specific resonance responses to the patient [8]-[13]; and other. In her review on the information medicine, Sagi [14] gives evidence that correcting the flaw in the information can be more effective than interfering with the biochemical processes resulting from the flawed information.

Fröhlich [15] was probably the first who proposed the project "Theoretical Physics and Biology", and he developed an idea of long-range phase correlations, i.e., coherence, which had been found to be a general concept required in the description of a nontrivial (non-spatial) order as in living organisms. He considered such hypotheses as 1) energy pumping to excite organs at correct frequency, 2) membrane deformations stimulating cell division, 3) growth inhibition when cell density suppresses coherent modes, 4) absorption defining range of phase correlations, etc. $\mathrm{He}$ combined high frequencies of electromagnetic fields and cooperative behaviour of the system studied with long-range phase correlations and applied this to biological systems because similar effects were observed in pure physical condensed matter systems.
Smith [16]-[19], following Fröhlich's ideas, showed that in the water studied homeopathy forms a domain of coherent frequency through long-range order, which means that water has a memory. In addition, Smith investigated the electromagnetic response of various biosystems and organs to an external signal in a wide frequency range, $10^{-4}$ to $10^{9}$ $\mathrm{Hz}$, and concluded that distorted "fractal" signals in the systems under consideration primarily attack the parasympathetic nervous system - this system covers a peculiar network cocoon of each organ and controls its functioning; if failures in the parasympathetic nervous system begin, it is automatically transferred to a particular organ or system of the body, creating specific links.

In the reverse Voll method, researchers [8]-[13] studied for the ability to induce an effect on the body that would correct a particular organ. Transmitting certain field signals, which carry peculiar codes to each diseased organ for its proper operation, carries out exposure to the body. This approach has successfully been applied for the treatment of almost all diseases (cancer, dementia, senile infirmity, gangrene, lack of growth hormone in the child, etc.), since the field signals are the correct frequency(ies) of functioning of organs in their healthy range, which are primarily a fundamental aspect of nature, not distorted ones (or "fractal" according to C.W. Smith) that manifest themselves in a diseased state. Information therapy adjusts the frequencies at which organ systems work, and therefore treats the diseases themselves eliminating the primary source of their occurrence. In his co-authored review, Gariaev [20] 
describes a theory of wave genetics, which was experimentally proved; namely, it was demonstrated the existence of wave genes in the form of actual text-like structures and mobile holographic constructions chromosome continuum. The authors mention that the human body, its cells, can understand the wave in two senses - safe and dangerous. They note that the chromosomes emit sound and coherent photons, which are converted into radio waves, and then to the real level of encoding genetic information to the wave, complementing real; these real and wave series genome are involved in the management of the body's metabolism and in its embryonic development. Is not Gariaev's research a confirmation of Bounias' [21] ideas about the connection between matter and spirit?

We have also to mention Puthoff and Targ [22], [23] who initiated theoretical, experimental and operational programmes in biofeedback and biofield measurements. Later their work resulted [24] in the study of psychokinesis, i.e. the direct influence of mind on matter ("psi", "paranormal", or "parapsychology") without the presence of extraneous physical fields and devices. That was the Remote Viewing programme, which involved precognition and clairvoyance where the practitioner receives information regardless of distance and time.

In the present work, we reveal the physical nature of field signals capable of correcting the functioning of organs. These field signals are not related to the electromagnetic field but are mass carriers that cause short-range interaction between particles, that is, they are carriers of quantummechanical interaction. These carriers were named inertons [25] since they arise because of the resistance of space to the motion of any material object (i.e., inertons are related to the force of inertia). The absorption of inertons (i.e., extra mass) by the system immediately changes the force of interaction between the particles, and therefore changes the electromagnetic signals induced by the system.

\section{MATERIALS AND METHODS}

\section{A. Phonons in the Crystal Lattice}

Materials that can be used in the practice of information medicine are metallic plates and chips, and organic dielectric substances. The investigation of information signals we carried out by the methods of theoretical physics. Vibrations of entities and the physical or biophysical system in question are modelled by so-called optical and acoustic phonons.

In a metal, electron scattering by lattice vibrations is the main scattering mechanism. Let the wave vectors $\vec{k}$ and $\vec{k}^{\prime}$ describe the states of the electron before and after scattering, and let the wave vector $\vec{q}$ describe the collective state of the lattice (i.e., the phonon). Laws of conservation of momentum and energy are:

$$
\begin{aligned}
& \hbar \vec{k}^{\prime}=\hbar \vec{k} \pm \hbar \vec{q}, \\
& \hbar \vec{k}^{\prime 2} /\left(2 m^{*}\right)=\hbar \vec{k}^{2} /\left(2 m^{*}\right) \pm \hbar \omega_{\vec{q}}
\end{aligned}
$$

where $\omega_{q}=v_{s} q, \quad v_{s}$ is the speed of sound, \pm means absorption (+) and emission (-) of the phonon. From these equations:

$$
q= \pm 2 k \cos \theta
$$

where $\theta$ is the angle between the directions of vectors $\vec{k}$ and $\vec{q}$.

The electrons in copper at room temperature are characterised by the parameters: de Broglie's wavelength $\lambda_{\mathrm{dB}}=4.5 \times 10^{-10} \mathrm{~m}$, the wave number $k=1.48 \times 10^{10} \mathrm{~m}^{-1}$ and the frequency $\nu=\nu_{\mathrm{F}} / \lambda_{\mathrm{dB}}=3.69 \times 10^{14} \mathrm{~Hz}$. We can impose on the system under study a set of vibrations that can be denoted by the wave numbers $q_{i}(i=1,2,3, \ldots)$; the corresponding values of $\cos \theta_{i}$ can be small so that all wave numbers of the corresponding phonons will satisfy the inequalities $q_{i}<<k$. So, in copper, we need to be interested in phonons with cyclic frequencies $\omega_{q}<<\omega_{\mathrm{D}}=7.144 \times 10^{13} \mathrm{~s}^{-1}\left(\omega_{\mathrm{D}}\right.$ is the Debye frequency) and the wave numbers $q<<1.5 \times 10^{10} \mathrm{~m}^{-1}$.

The average phonon number depends on the temperature $T$ and the frequency of the phonons $\omega_{q_{i}}$ as the BoseEinstein statistics prescribes:

$$
\left\langle n_{i}\right\rangle=\frac{1}{\exp \left[\hbar \omega_{q_{i}} /\left(k_{\mathrm{B}} T\right)-1\right]}
$$

For acoustic phonons $\left(\omega_{q}<<\omega_{\mathrm{D}}\right)$ :

$$
\left\langle n_{i}\right\rangle=k_{\mathrm{B}} T /\left(\hbar \omega_{q_{i}}\right)
$$

For example, for phonons with cyclic frequencies $2 \pi \cdot\left(10^{6}-10^{12}\right) \quad \mathrm{s}^{-1}$ at room temperature their average number is $\left\langle n_{i}\right\rangle \approx 6.35 \times\left(10^{6}-10^{12}\right)$. So, the number of acoustic phonons is huge.

These phonons are in thermal equilibrium with the environment - at the edges of the system they interact with the thermal oscillations of the thermostat (air, other physical systems, etc.).

\section{B. Inertons in the Crystal Lattice}

In our studies [25] it was shown that quantum mechanics could be deduced from submicroscopic mechanics, which follows directly from the theory of the structure of real physical space. Physical space is a mathematical lattice of primary topological balls with a size estimated as Planck's length $\ell_{\mathrm{P}}=\sqrt{\hbar G / c^{3}} \simeq 1.62 \times 10^{-35} \mathrm{~m}$; this lattice Michel Bounias [26] named a tessellattice. Therefore, the tessellattice is a primary fundamental physical substrate, which must be characterised by its elasticity and speed of sound (the speed of light $c$ ). Matter is created from a cell of the tessellattice at a volumetric fractal deformation, which 
corresponds to the appearance of mass. For example, an electron is a volumetrically deformed topological ball, i.e., a fractally contracted cell of the tessellattice, which moves squeezing through between ongoing cells and therefore interacts with them.

When such a particle moves due to a collision with cells of the real space, the particle mass defragments so that volumetric fractals (i.e., wrinkles of the ball) break off from the particle and go apart as quasiparticles with a speed $\vec{c}+\overrightarrow{\dot{x}}$ ( $\vec{c}$ is the velocity in the transverse direction, and $\overrightarrow{\dot{x}}$ is the velocity in the direction of particle movement). Quasiparticles, which appear at the collisions of the particle with ongoing cells of the tessellattice, are inertons [25]. Inertons move according to the relay mechanism hopping from cell to cell and they are mass carriers, that is, they carry volumetric fractals, which are fragments of the particle mass. They go away at a distance $\Lambda$ from the particle and so the tessellattice is elastic, it reflects the inertons back to the particle.

Having passed the distance $\lambda_{\mathrm{dB}}=h /(m v)$, the particle completely drops all volume fractal deformations (wrinkles) that go to the radiated inerton cloud, and the particle goes into the state of tension $\vec{\xi}$. In this state, its volume returns to its original volume, as in a degenerate cell of the tessellattice, but at the same time, the cell goes into a tense state, that is, becomes rigid, and the vector indicates the direction of tension along the particle path. And in the following segment $\lambda_{\mathrm{dB}}=h /(m v)$, the inertons return to the particle and transmit the deformation fragments, i.e., they guide the particle (as de Broglie pointed out: the real wave should guide the particle). Therefore, the particle again gains its mass - the centre cell once more becomes contracted by volumetric fractals (wrinkles); and so on.

Thus, any mass particle (electron, atom, etc.) moves wrapped in its inerton cloud and the volume of this cloud is:

$$
\lambda_{\mathrm{dB}} \times \pi \Lambda^{2}
$$

where $\Lambda=\lambda_{\mathrm{dB}} c / v$ is the amplitude of the inerton cloud.

Since the particle's mass is a variable parameter (owing to the interaction of the particle with ongoing cells of the space) [25], the mass in a rectilinear motion or the mass of atoms that oscillate forming acoustic waves (phonons), can be described by the following Lagrangian:

$$
L=\frac{1}{2} \lambda^{2} \dot{m}^{2}+\frac{1}{2} m_{0}^{2} \dot{\vec{\xi}}^{2}+v m_{0} \lambda \dot{m} \nabla \vec{\xi}
$$

where the variables $m(\vec{x}, t)$ and $\vec{\xi}(\vec{x}, t)$ characterise the mass and tension of the particle, respectively. In the expression (5) $\lambda$ is the characteristic particle wave, (the de Broglie wavelength in the case of an electron or the wavelength of an acoustic phonon in the case of the crystal lattice); $m_{0}$ is the initial mass of the particle (electron or phonon) and $v$ the initial velocity of the particle (or the velocity of the acoustic wave, i.e. phonons). The Euler-
Lagrange equations for variables $m$ and $\vec{\xi}$ are (since in the Lagrangian (5) we have $\nabla \vec{\xi}$ ) [27].

$$
\begin{aligned}
& \frac{\partial}{\partial t}\left(\frac{\partial L}{\partial \dot{Q}}\right)-\frac{\delta L}{\delta Q}=0, \\
& \frac{\delta L}{\delta Q}=\frac{\partial L}{\partial Q}-\frac{\partial}{\partial x} \frac{\partial L}{\partial(\partial Q / \partial x)}-\frac{\partial}{\partial y} \frac{\partial L}{\partial(\partial Q / \partial y)}-\frac{\partial}{\partial z} \frac{\partial L}{\partial(\partial Q / \partial z)}
\end{aligned}
$$

(where $Q \equiv m, \vec{\xi}$ ), lead us to the following differential equations in explicit form.

$$
\lambda^{2} \ddot{m}+v m_{0} \lambda \vec{\nabla} \dot{\vec{\xi}}=0, \quad m_{0}^{2} \ddot{\vec{\xi}}+v m_{0} \lambda \vec{\nabla} \dot{m}=0
$$

From these equations we obtain typical wave equations of motion for each of the two variables:

$$
\ddot{m}-v^{2} \nabla^{2} m=0, \ddot{\vec{\xi}}-v^{2} \nabla^{2} \vec{\xi}=0
$$

Therefore, the equations for the variation of the mass $m$ and tension $\vec{\xi}$, which move in antiphase, show that any matter moves as a typical wave. In such a wave the fractal contraction (the mass $m$ ) is periodically replaced by the stretching (the tension $\vec{\xi}$ ).

In the crystal lattice atoms oscillate in acoustic waves. The inerton clouds of the individual atoms overlap and, therefore, the space between the atoms is filled with inertons and these inertons oscillate in tact with the motion of the atoms. Therefore, like the vibrations of atoms, inertons have a large set of harmonics. Thus, in the crystal, every $i$ th phonon mode is filled with inertons, which can be regarded as an inerton gas in some approximation. Indeed, the distance between atoms in the crystal is approximately $g=0.3 \mathrm{~nm}$ and this space is filled with an inerton gas having up to $10^{25}$ quasiparticles (for the minimum wavelength this number is $\left.2 g / \ell_{\mathrm{P}} \sim 10^{25}\right)$. It looks like the phonon is just a wave casing and the filler is the inertons.

When the phonons at the edge of the crystal lattice transmit their vibrational energy to the environment, such as the air, the atoms of the crystal transmit acoustic vibrations to the air molecules. At the same time, the inerton subsystem, which accompanies the phonons, extends beyond the crystal, where they must be absorbed by surrounding physical systems. But the free path of the radiated inerton wave can be very long. It can migrate in space until it is absorbed by the physical system with its corresponding resonant parameters.

In general, the vibrations of the crystal lattice and the processes of electron scattering by these oscillations are in thermal equilibrium with the environment. However, the presence of the inerton subsystem in the oscillatory process of the lattice allows one to establish true long-lived states that will derive a set of certain oscillatory modes out of the Planck thermal distribution (3). In other words, the inerton subsystem makes it possible to control the population of certain oscillatory modes in a condensed medium. 
Let us consider the conditions of emanation of inerton waves and their absorption by another crystal, so that the inertons are not scattered in the absorber crystal with exactly the same parameters that they had during emanation. To do this, Lagrangian (5) for variables $m(\vec{x}, t)$ and $\vec{\xi}(\vec{x}, t)$ needs to be complemented by two members providing resonant emission and absorption (the arrival and outflow of contraction and tension, i.e. $m$ and $\vec{\xi}$, which the inertons transfer):

$$
L=\frac{1}{2} \lambda^{2} \dot{m}^{2}+\frac{1}{2} m_{0}^{2} \dot{\vec{\xi}}^{2}+v m_{0} \lambda \dot{m} \nabla \vec{\xi}+m G(\vec{x}, t)+\vec{\xi} \vec{F}(\vec{x}, t)
$$

In this case, the Euler-Lagrange equations look like nonlinear wave equations:

$$
\begin{aligned}
& \ddot{m}-v^{2} \nabla^{2} m+\int^{t} \vec{\nabla} \vec{F}\left(\vec{x}, t^{\prime}\right) d t^{\prime}=0 \\
& \ddot{\vec{\xi}}-v^{2} \nabla^{2} \vec{\xi}+\int^{t} \nabla G\left(\vec{x}, t^{\prime}\right) d t^{\prime}=0
\end{aligned}
$$

Equations (9) and (10) are similar and have solutions that differ only in phase shift by $\pi / 2$. Therefore, we will only consider equation (9).

In Eq. (9) let us allow the separation of the variable $m(\vec{x}, t)=M(\vec{x}) \vartheta(t)$ and also we can put $\int^{t} \vec{\nabla} \vec{F}\left(\vec{x}, t^{\prime}\right) d t^{\prime}=f(\vec{x}) e^{i \omega t}$. Then from equation (9) we obtain the inhomogeneous Helmholtz equation:

$$
\Delta M(\vec{x})+q^{2} M(\vec{x})=f(\vec{x})
$$

where $q^{2}=\omega^{2} / v^{2}$ is the square of the wave vector module.

Let all the functions in equation (11) be dimensionless. Fundamental solutions of the Helmholtz operator in 3D space at $f(\vec{x})=\delta(\vec{x})$ (see, for example, Ref. [28]) are:

$M(\vec{x})=-\frac{e^{i q|\vec{x}|}}{4 \pi|\vec{x}|}, M^{*}(\vec{x})=-\frac{e^{-i q|\vec{x}|}}{4 \pi|\vec{x}|}$

The Fourier transform method reduces Eq. (11) to an algebraic equation:

$$
\left(-\mu^{2}+k^{2}\right) f[M(\vec{x})]=1
$$

This transformation makes it possible to find the Helmholtz operator solution in 2D space in the class of Hankel functions [28].

$$
\begin{aligned}
& M(q|\vec{x}|)=-\frac{i}{4} H_{0}^{(1)}(q|\vec{x}|), \\
& M^{*}(q|\vec{x}|)=\frac{i}{4} H_{0}^{(2)}(q|\vec{x}|)
\end{aligned}
$$

Interestingly, in the case of a sharp discontinuity of a long plate or curve, both sides of the discontinuity can also be described [29] by Hankel functions (14).

For large values $q|\vec{x}|$ (i.e. $q|\vec{x}|>>1$ ), the following asymptotic representation of Hankel functions can be used [30]:

$$
\begin{aligned}
& H_{0}^{(1)}(q|\vec{x}|) \simeq \sqrt{\frac{2}{\pi q|\vec{x}|}}(\cos \varphi+i \sin \varphi), \\
& H_{0}^{(2)}(q|\vec{x}|)=H_{0}^{(1)^{*}}(q|\vec{x}|)
\end{aligned}
$$

where

$$
\varphi=q|\vec{x}|-\left.\frac{\pi \cdot(\nu+1 / 2)}{2}\right|_{\nu=0}
$$

Then two solutions (14) take the form:

$$
\begin{aligned}
& M_{1}(q|\vec{x}|) \simeq-\frac{i}{4} \sqrt{\frac{2}{\pi q|\vec{x}|}}(\cos \varphi+i \sin \varphi), \\
& M_{2}(q|\vec{x}|) \simeq \frac{i}{4} \sqrt{\frac{2}{\pi q|\vec{x}|}}(\cos \varphi-i \sin \varphi)
\end{aligned}
$$

The solutions (17) determine the mass of the inerton wave at the point $\vec{x}$ of the metal plate. Obviously, the solution to the mass must be real and positive, and this is possible only with certain values $\varphi=\varphi_{\text {real }}$. True solutions to the mass have the form:

$M_{1}(q|\vec{x}|)=M_{2}(q|\vec{x}|) \simeq \frac{1}{4} \sqrt{\frac{2}{\pi q|\vec{x}|}} \sin \varphi_{\text {real }}$

The solution for the tension of the inerton wavelet, which is in antiphase to the solution (18), looks similar:

$$
\Xi_{1}(q|\vec{x}|)=\Xi_{2}(q|\vec{x}|) \simeq \frac{1}{4} \sqrt{\frac{2}{\pi q|\vec{x}|}} \cos \varphi_{\text {real }}
$$

Obviously, for the emanation of the inerton wavelet from the edge of the plate, antinode should be established at this edge. That is $\sin \varphi_{\text {real }}=0$ and $\cos \varphi_{\text {real }}=1$. Then at the edge of the plate the mass of the inerton wavelet becomes $M_{1}(q|\vec{x}|)=M_{2}(q|\vec{x}|)=0$, and the tension:

$\Xi_{1}(q|\vec{x}|)=\Xi_{2}(q|\vec{x}|) \simeq \frac{1}{4} \sqrt{\frac{2}{\pi q|\vec{x}|}}$

This means that the product of the wave number $q$ and the modulus of the radius vector $|\vec{x}|$ (where $\vec{x}$ is the edge of the 2D plate) imply the following conditions: 


$$
\varphi_{\text {real }}=q|\vec{x}|-\frac{\pi}{4}=2 \pi l, l=0,1,2, \ldots
$$

that is

$$
q|\vec{x}|=2 \pi l+\frac{\pi}{4}, l=0,1,2, \ldots
$$

Thus, we have shown that inerton wavelets indeed can be emanated from a solid.

\section{RESULTS}

\section{A. Estimation of Inerton Intensity}

The vibrational frequencies of atoms, which give a large average value of excited phonons (3), lie in the frequency range $\omega=2 \pi \cdot\left(10^{6}-10^{12}\right) \mathrm{s}^{-1}$. Then, for a copper / brass plate, the corresponding wavelengths lie in the range of $\lambda=3 \cdot\left(10^{-8}-10^{-2}\right) \mathrm{m}$, and the appropriate value of the wave number is $q=2 \pi / \lambda$.

A nonzero solution (20), (22) means that there is an inflow or outflow of inerton wavelets (in a state of the tension) at the edge of the plate at the wavelength $\lambda$ and frequency $\nu$ specified in Table I.

The presence of inflow / outflow of inerton wavelets in the system under study changes the Planckian distribution of phonons (3), (4). At the indicated wavelengths $\lambda_{l}$ and frequencies $\nu_{l}$ (Table I), the distribution of phonons in the plate already has a different population, namely expression (4) changes to:

$$
\left\langle n_{l}\right\rangle=k_{\mathrm{B}} T /\left(\hbar \omega_{q_{l}}\right)+\delta n_{l}
$$

where $\delta n_{l}$ has the order of the thermal distribution (4) because the inerton generation itself is due to the thermal field. So, in the first approximation, we can assume that distribution (23) has the form $\left\langle n_{l}\right\rangle \approx 2 k_{\mathrm{B}} T /\left(\hbar \omega_{q_{l}}\right)$, i.e. $\left\langle n_{l}\right\rangle \approx 2 k_{\mathrm{B}} T /\left(\hbar \omega_{l}\right)$ and then the population at the selected $l$ th levels will reach $10^{7}-10^{11}$ phonons, whose inner body is filled with inertons.

TABLE I: THE ARRANGEMENT $\lambda$, FREQUENCY $\nu$, AND RELATIVE

\begin{tabular}{|c|c|c|c|}
\hline$l$ & $\lambda_{l}(\mathrm{~m})$ & $v_{l}(\mathrm{~Hz})$ & $\begin{array}{c}\Xi_{l} \\
\text { (Expression (20)) }\end{array}$ \\
\hline 1 & $1.6 \times 10^{-2}$ & $3.264 \times 10^{5}$ & $7.5 \times 10^{-2}$ \\
\hline 10 & $1.77 \times 10^{-3}$ & $2.655 \times 10^{6}$ & $2.495 \times 10^{-2}$ \\
\hline $10^{2}$ & $1.798 \times 10^{-4}$ & $2.614 \times 10^{7}$ & $7.953 \times 10^{-3}$ \\
\hline $10^{3}$ & $1.8 \times 10^{-5}$ & $2.611 \times 10^{8}$ & $2.516 \times 10^{-3}$ \\
\hline $10^{4}$ & $1.8 \times 10^{-6}$ & $2.611 \times 10^{9}$ & $7.958 \times 10^{-3}$ \\
\hline $10^{5}$ & $1.8 \times 10^{-7}$ & $2.611 \times 10^{10}$ & $2.516 \times 10^{-4}$ \\
\hline $10^{6}$ & $1.8 \times 10^{-8}$ & $2.611 \times 10^{11}$ & $7.958 \times 10^{-5}$ \\
\hline $10^{7}$ & $1.8 \times 10^{-9}$ & $2.611 \times 10^{12}$ & $2.516 \times 10^{-5}$ \\
\hline
\end{tabular}
AMPLITUDE OF THE MODULUS OF THE TENSION $\Xi$ (20) OF THE INERTON WAVELET GIVEN BY THE NUMBER $L(22)$

Thus, at these frequencies, the transmission of phonon energy occurs: at the output of the plate, the phonon casing remains in the system of the lattice vibrations, but the internal inerton subsystem goes out in the form of an inerton wavelet. The amplitude of this wavelet is of the order of thermal energy, but the wavelet coming out of the plate is not thermalised, because the permeability of inertons is very high. That is, the inerton wavelet does not interact with the environment until it encounters a physical system with the same parameters. In other words, precise resonance is required to absorb this inerton wavelet.

\section{B. Medical Application}

A receiver of inerton wavelets must have the same boundary conditions as the emitter plate. For example, two pieces of a plate cut or broken in the middle become an emitter and a receiver, respectively, because they have the same boundary conditions. Of course, an inerton wavelet should decay or dissipate over time, but its lifetime can be quite long (by our measurements [25], nonequilibrium inerton states in macroscopic soft systems live for several days). And during this time, a free inerton wavelet is able to travel many thousand or even million kilometres (at a speed possibly higher than $c$ ).

With distance, the intensity of the inerton wavelets generated by the plate has to decrease in accordance with the inverse-square law:

$\left\langle n_{l}(R)\right\rangle=\left\langle n_{l}\right\rangle \frac{\sigma}{4 \pi R^{2}}$

where $\left\langle n_{l}(R)\right\rangle$ is the number of wavelets with index $l$ that comes to the distance $R$ from the emitting source; $\left\langle n_{l}\right\rangle \approx 2 k_{\mathrm{B}} T /\left(\hbar \omega_{l}\right)$ is the average number of inerton wavelets. The receiver will absorb the following number of waves in $1 \mathrm{~s}$ :

$I_{l}(R)=\left\langle n_{l}\right\rangle \nu_{l} \frac{\sigma}{4 \pi R^{2}} \approx \frac{k_{\mathrm{B}} T \sigma}{4 \pi h R^{2}}$

In our work on information therapy, we use small plates/chips made of copper and brass. The size of the absorber plate is $18 \mathrm{~mm} \times 18 \mathrm{~mm} \times 0.230 \mathrm{~mm}$ and then the average surface area of the receiver is $\sigma \approx 1.77 \times 10^{-5} \mathrm{~m}^{2}$. Table II shows the order of magnitude of the inerton wavelet intensity $I_{l}(R)$ at different distances.

TABLE II: THE INTENSITY (25) OF THE INERTON SIGNAL WITH ANY OF THE $L T H$ FREQUENCIES AT A DISTANCE $R$ FROM THE SOURCE.

\begin{tabular}{ccc}
\hline$R$ & $I_{l}(R)$ & $I_{l}(R) \times$ day \\
$(\mathrm{m})$ & $\left(\mathrm{s}^{-1}\right)$ & $($ i.e., per 24 hours $)$ \\
\hline 1 & $10^{7}$ & $10^{12}$ \\
10 & $10^{5}$ & $10^{10}$ \\
$10^{2}$ & $10^{3}$ & $10^{8}$ \\
$10^{3}$ & 10 & $10^{6}$ \\
$10^{4}$ & $10^{-1}$ & $10^{4}$ \\
$10^{5}$ & $10^{-3}$ & $10^{2}$ \\
$10^{6}$ & $10^{-5}$ & 1 \\
$10^{7}$ & $10^{-7}$ & $10^{-2}$ \\
\hline
\end{tabular}

As can be seen from Table 2, the information signals, i.e. inerton wavelets, reach the receiver plate at a distance of $1-$ $10 \mathrm{~km}$ every second. In the long run, information influence 
is also established, but over time. For a distance of 10,000 $\mathrm{km}$, it takes up to two days to bring an informational message.

Inerton information signals can be modulated. That is, a monoinerton wavelet can leave the emitter with an additional set of frequencies. Such wavelets will deliver to the receiver the information necessary for introduction into a diseased organism, i.e., a set of certain frequency modes, which carry information to a specific system of the organism in order to improve its functioning and, possibly, even restore almost completely to a healthy state. As can be seen from Table II, at relatively short distances, informational inerton signals quite often come to the receiver, and therefore to the patient who needs treatment. But at distances of thousands of kilometers, the number of corrective signals needed by the body decreases sharply. However, these signals do come regularly.

Paper [31] describes a method for measuring lowintensity signals, and even those that are significantly lower in amplitude than the noise level; we detected even signals that were 10,000 times weaker than the noise level. However, the signals extracted from the noise should be repeated periodically. In the case of emission of inerton wavelets, it can be said with certainty that this is a periodic process and therefore they will get to the remote receiver regularly, albeit with some delay in time. Therefore, these wavelets will still bring the necessary information to the patient's body.

In addition, we observe that a continuous connection (a kind of a complexity) is established between the emitter and the receiver. Indeed, we can examine the patient's body at a distance of many kilometers in real time. How is this physically possible? A brass chip used has about $\aleph \sim 10^{19}$ atoms. A spectrum of acoustic wavelegths can easily be estimated for 1D approximation by a relationship $\lambda_{n}=2 \mathrm{an}$ where $a$ is the lattice constant and $n=2,3, \ldots, \aleph$. Then the spectrum of the appropriated inertons becomes $\Lambda_{n}=\lambda_{n} c / v_{\text {sound }}$ (see Ref. [25], Ch. 8. Gravity). The fundamental inerton standing wavelegth for such small brass plate is $\Lambda_{\aleph} \sim 10^{15} \mathrm{~m}$, which means that the connection between the emitter and the receiver must exist even when they are located in diametrically distant points on the surface of our planet. Moreover, the emitter and the receiver may experience each other even in the case when the emitter is on the Earth and the receiver is on the Moon.

An information therapist uses an electronic device named "INTERA-DiaCor" (the Ukrainian State Registration Certificate No. 3277/2004 of October 30, 2009) connected to the computer with the necessary software. The device has a certain matrix, a database of thousands of signals recorded from healthy and diseased organs and systems of the human body, which were averaged over tens of thousands of people. After a thorough examination of the patient by the methods of information therapy, i.e. analysis of signals received from the systems of his/her body, the therapist determines a set of corrective signals and records them in a droplet of a material, which could be a melted wax or tin. This material is dropped on a brass plate to which the set on needed signals is transferred. After solidification, the material has frozen certain vibrational modes, which are characterised by higher intensities than the Planck distribution (3) and (4) prescribes. Then the droplet(s) are attached to the emitter plate and the latter transmits the signals to a receiver plate fasted to the patient's body by the mechanism described in this article.

Statistics collected from recovering patients show the high efficiency of the information therapy method (or the inerton information therapy). A description of treatment protocols and results will be reported in another publication. Here we give only brief information in relation to a few cases.

A year ago, one of us (VK) was examined by the methods of information therapy and a set of correcting signals was recommended. The emitter plate was constantly in the office of the practicing doctor, and the patient $(\mathrm{VK})$ was in motion at an average distance of $10 \mathrm{~km}$ from the office. In a month after wearing of a brass plate (the receiver chip described above), the vision of the right eye has almost returned to normal. Although, after 2000, floating dark flies appeared, which by mid-2019 had already shaded around up to one third of the field of view. Removing dark spots indicate to an introduced correction to the optic nerve, which was done via information inerton wavelets.

On August 18, 2020 a patient (a physicist, 27 y.o. living in Eindhoven, the Netherlands) attached to his arm a brass plate and the therapist sitting in Kyiv, Ukraine, examined the patient's body. Then a set of codes was prescribed and launched to the patient via the inerton channel. The distance between the two cities is about $1700 \mathrm{~km}$. The second session was on October 3, 2020. All the systems of the patient's body became functioning better circa $30 \%$. It is interesting that before the second session the patient informed us that he stopped to smoke cannabis; that is, he does not have interest to do that. This is because the patient's physiological and psychological conditions significantly improved.

One more patient (a housewife, 63 y.o. living in the village of Satanivka, Cherkasy region, Ukraine, $210 \mathrm{~km}$ from the city of Kyiv) had a diagnosis as below: dyscirculatory encephalopathy, vertebrobasilar insufficiency, osteochondrosis of the cervical spine, disc protrusion into the spinal canal in segments C4-C5, C5-C6, C6-C7. The patient was informed of the onset of Parkinson's disease. She was unable to do any work at the family's garden and at home, she could not walk along straight line at all. On June 24, 2020 the first session of remote information therapy was performed. The next session was on July 23, 2020. In August, the patient looked as practically healthy she began to easily walk along straight line and started to do any work both at home and the garden. The third session was conducted on October 20, 2020. Since that the patient feels even better.

\section{CONCLUSION}

The physical theory and results presented in this paper are quite new. Nevertheless, we have shown one more successful application of the recent finding in fundamental physics: information therapy based on the transmission of correct inerton signals (inerton wavelets) can be surveyed as 
one of the most powerful methods of healing. The practice of inerton information therapy could be an excellent tool for both early diagnosis and successful treatment of completely different diseases, including the coronavirus or any chronic diseases of uncertain origin. Moreover, the wireless inerton communication channel provides diagnostics of the patient at a distance at any time with the further forwarding of tweaked signals for more accurate correction of the body's diseased systems.

It the further studies, it will be interesting to try to collect a wider database of signals of different body systems including those that could characterise optical vibrational spectra. Could inerton-photon wavelets transmit information stored in such spectra? If yes, how one can transmit such signals and what may be the average path length of inertonphoton wavelets? These issues require a separate consideration.

The described approach does not require the informed consent of the patient, which is currently a cornerstone of ethics in clinical trials. This means that information therapy is a step forward in comparison with pharmacological medicine because it does not have any molecular agent or destructive radiation, which can adversely affect the human body.

Finally, the obtained results allow us to run a channel of inerton radio communication, which can obviously compete seriously with existing photon (i.e. electromagnetic) radio communication.

\section{REFERENCES}

[1] V. Krasnoholovets, S. Skliarenko and O. Strokach, "On the behavior of physical parameters of aqueous solutions affected by the inerton field of Teslar® Technology", Int. J. Modern Phys. B, vol. 20, no. 1, pp. 1-14, 2006 (also arXiv:0810.2005).

[2] V. Krasnoholovets, S. Skliarenko and O. Strokach, "The study of the influence of a scalar physical field on aqueous solutions in a critical range", J. Molecular Liquids, vol. 127, nos. 1-3, pp. 50-52, 2006.

[3] E. Andreev, G. Dovbeshko, and V. Krasnoholovets, "The study of influence of the Teslar technology on aqueous solution of some biomolecules," Research Letters in Physical Chemistry, Vol. 2007, Article ID 94286, 5 pages, 2007 (also arxiv:1204.6062).

[4] E. A. Andreyev, M. U. Bilyi, and S. P. Sit'ko, "The reaction of the human body to electromagnetic radiation of the millimetre range," Bulletin of the USSR Academy of Sciences, no. 1, pp. 24-33, 1985; in Muscovite.

[5] S. P. Sit'ko, E. A. Andreyev, L. N. Christophorov, and A. A. Serikov, "Research into the physical mechanisms of low-intensity microwave radiation upon biological systems," I. Preprint ITP-90-49-E, Kyiv, 1990, 28 p.

[6] D. Rakoviĉ, Z. Jovanović-Ignjatić, D. Radenović, M. Tomašević, E. Jovanov, V. Radivojević, Ž Martinović, P. Šuković, M. Car, and L. Škarić, "An overview of microwave resonance therapy and EEG correlates of microwave resonance relaxation and other consciousness altering techniques," Electro- and Magnetobiology, vol. 19, no. 2, pp. $195-222,2000$

[7] A. Oliveira, "Electroacupuncture according to Voll: historical background and literature review," J. Acupuncture and Oriental Medicine, Winter 2016, pp. 5-10.

[8] Z. D. Skrypnyuk, "Information and negentropic therapy," Information and Negentropic Therapy. Ed.: Z. D. Skrypniuk, Kyiv, Volume 1994, no. 1, pp. 4-8; in Muscovite.

[9] Z. D. Skrypnyuk, "Basic concepts of traditional Chinese medicine from the point of view of an informotherapist," Information and Negentropic Therapy, Ed.: Z. D. Skripniuk, Kyiv, Volume 1994, no. 1, pp. 19-24; in Muscovite.

[10] V. Ya. Kobyliansky, "Microgenerator informotherapy as a means of restoration of functional activity of information channels," Information and Negentropic Therapy, Eds.: V. Ya. Kobyliansky, R.
M. Kobylianska, Z. D. Skrypniuk, and K. I. Shokaliuk, Kyiv, Special Volume 1999, pp. 60-62.

[11] Z. D. Skrypniuk, "Strategy and tactics of choice of diagnostics and therapy in general and family medicine," Information and Negentropic Therapy, Ed.: Z. D. Skripniuk, Kyiv, Volume 2001, pp. 133-134; in Ukrainian.

[12] Z. D. Skripniuk, "Phonetics, morphology and syntax of cell languages," Information and Negentropic Therapy, Ed: Z. D. Skripniuk, Kyiv, Volume 2001, pp. 135-136; in Ukrainian.

[13] V. M. Fedorivskyi, and Z. D. Skrypniuk, "Comparative characteristics of the action of the micro generator "BEST" and its electronic version," Information and Negentropic Therapy, Ed.: Z.D. Skrypniuk, Kyiv, Volume 2014, pp.115-139; in Ukrainian.

[14] M. Sagi, "The new homeopathy: A new paradigm in information medicine," World Futures: The Journal of New Paradigm Research, vol. 72, nos. 3-4, pp. 53-68, 2016.

[15] H. Fröhlich, "Long range coherence and energy storage in biological systems," Int. J. Quantum Chem., vol. 2, pp. 641-649, 1968.

[16] C. W. Smith, "Coherence in living biological systems," Neural Network World, vol. 3, pp. 379-388, 1994.

[17] C. W. Smith, "Can homeopathy ameliorate ongoing sickness?" The Journal of Alternative and Complementary Medicine, vol. 15, no. 5, pp. $465-467,2009$.

[18] C. W. Smith, "Reflected light modulated by bio-fields," The Journal of Alternative and Complementary Medicine, vol. 16, no. 11, pp. 1133-1134, 2010.

[19] C. W. Smith, "Electromagnetic and magnetic vector potential bioinformation and water," Homeopathy, vol. 104, no. 4, pp. 301-304, 2015.

[20] P. P. Gariaev, and E. A. Leonova, "The strange world of wave genetics," DNA Decipher Journal, vol. 4, no. 1, pp. 39-56, 2014.

[21] M. Bounias, La Création de la vie: De la matière à l'esprit; Paris: Editions du Rocher, 1990.

[22] H. E. Puthoff and R. Targ, "PK experiments with Uri Geller and Ingo Swann," in Research in Parapsychology 1973, Roll, W. G., Morris, R. L. and Morris, J. D. eds., Scarecrow Press, Metuchen, New Jersey; pp. $125-128,1974$.

[23] H. E. Puthoff, and R. Targ, "Physics, Entropy and Psychokinesis," in Proc. Conf. Quantum Physics and Parapsychology (Geneva, Switz.), Parapsychology Foundation Publ., New York, 1975.

[24] E. W. Davis, "Teleportation Physics Study". Special Report. Air Fore Research Laboratory, Air Force Material Command, Edwards Air Force Base CA 93524-7048, 2004.

[25] V. Krasnoholovets, Structure of space and the submicroscopic deterministic concept of physics; Oakville, Canada, and Waretown, USA: Apple Academic Press, 2017.

[26] M. Bounias, and V. Krasnoholovets, "Scanning the structure of illknown spaces: Part 1. Founding principles about mathematical constitution of space," The Kybernetes: The International Journal of Systems and Cybernetics, vol. 32, no. 7/8, pp. 945-975, 2003 (also arXiv:0211096).

[27] D. ter Haar, Elements of Hamiltonian Mechanics; Moscow: Nauka, 1974, p. 173 (Muscovite translation from the Second edition, Pergamon Press, 1971).

[28] V. S. Vladimirov, and V. V. Zharinov, Equations of mathematical physics; Moscow: Physics and mathematics literature, 2000, p. 154; in Muscovite.

[29] A. M. Fridman, and A. V. Khoperskov, Physics of galactic disks; Moscow: Fizmatgid, 2011, p. 320; in Muscovite.

[30] A. Angot, Compléments de mathématiques à l'usage des ingénieurs de l'électrotechnique et des télécommunications; Paris: Masson, 1997, p. 381; in French.

[31] Yu. Zabulonov, V. Burtniak, and V. Krasnoholovets, "A method of rapid testing of radioactivity of different materials," J. Radiation Research and Applied Science, vol. 9, pp. 370-375, 2016. 


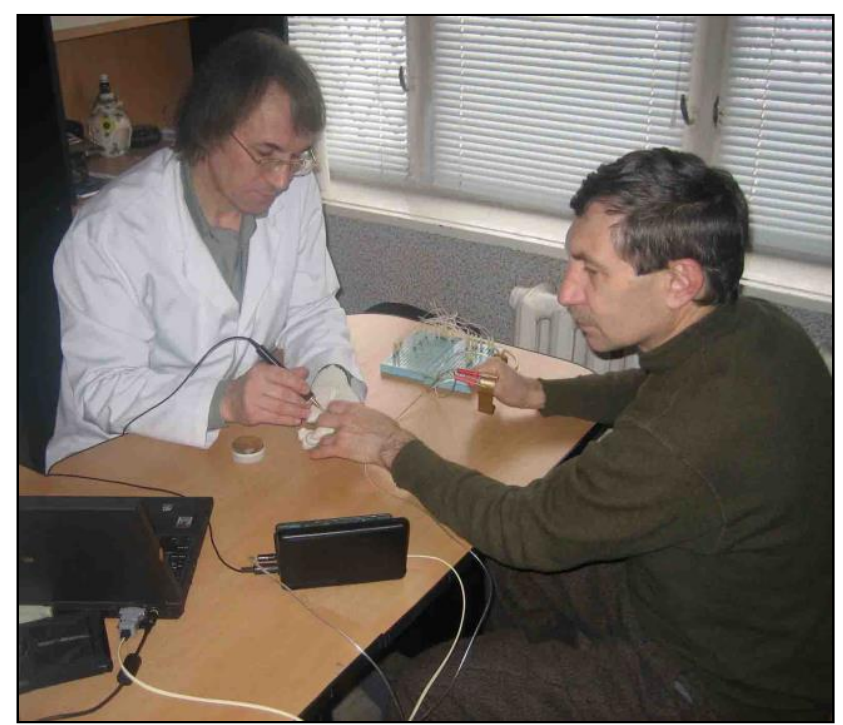

Vasyl Fedorivskyi (left) and Volodymyr Krasnoholovets (right)

V. Krasnoholovets was born in Kyiv, Ukraine. He graduated from a mathematical school, and then became a student of the Kyiv's Taras Shevchenko National University, Department of Physics, Faculty of Theoretical Physics; he received a master's degree in 1979. For next several years he worked as an experimentalist in the area of superconductivity at the Institute for Metal Physics, Kyiv. Since the beginning of 1980s and to now, he has been working at the Department of Theoretical Physics, Institute of Physics, Natl. Acad. Sci., Kyiv. Since 1993 he is a Senior Research Scientist. In parallel, he is involved in various activities associated with applied sciences.

V. Fedorivskyi was born in the Kmelnytsky region, Ukraine. In 1991 he graduated the Kyiv State Institute of Physical Culture, the Faculty of Rehabilitation. In 2009 he graduated from the Interregional Academy of Personnel Management, the Faculty of Psychology. He worked as a rehabilitation specialist in a private clinic for 13 years. He has been working as an information therapist for over 20 years and as a psychologist since 2009. 